Doug Geisler, Eva K. Grebel, and Dante Minniti, eds.

\title{
The NICMOS View of M31's Metal Rich Globular Clusters
}

\author{
Andrew W. Stephens \& Jay A. Frogel \\ The Ohio State University, Department of Astronomy, 140 West 18th \\ Avenue, Columbus, $\mathrm{OH}$ 43210, USA \\ Wendy Freedman \& Carme Gallart \\ Carnegie Observatories, USA
}

Pascale Jablonka

Observatoire de Paris-Meudon, France

Sergio Ortolani

Università di Padova, Italy

Alvio Renzini

European Southern Observatory, Germany

R. Michael Rich

University of California at Los Angeles, USA

\begin{abstract}
We have obtained HST-NICMOS observations of five of M31's most metal-rich globular clusters: G1, G170, G174, G177 \& G280. For the two clusters farthest from the nucleus, G1 and G280, we statistically subtract the field population and estimate metallicities using $K$ $(J-K)$ color-magnitude diagrams (CMDs). Based on the slopes of their infrared giant branches we estimate $[\mathrm{Fe} / \mathrm{H}]=-1.22 \pm 0.43 \mathrm{dex}$ for $\mathrm{G} 1$ and $-0.15 \pm 0.37$ dex for G280. We combine our infrared observations of G1 with two epochs of optical HST-WFPC2 $V$-band data and identify at least one LPV based on color and variability. The location of G1's giant branch in the $K,(V-K)$ CMD is very similar to that of M107, indicating a somewhat higher metallicity than our purely infrared CMD; $[\mathrm{Fe} / \mathrm{H}]=-0.9 \pm 0.2 \mathrm{dex}$.
\end{abstract}

\section{Introduction}

In Cycle 7 we proposed to obtain Hubble Space Telescope (HST) NICMOS $J H K$ images of 5 metal rich globular clusters in M31 (Program ID 7826). The clusters include G1, G170, G174, G177, and G280. Our observations were taken with the NICMOS camera 2 (NIC2), which has a plate scale of $\sim 0.0757^{\prime \prime}$ pixel $^{-1}$ and 
a field of view of $19.4^{\prime \prime}$ on a side $\left(376 \operatorname{arcsec}^{2}\right)$. Each of our targets was observed through three filters: F110W $(0.8-1.4 \mu \mathrm{m}), \mathrm{F} 160 \mathrm{~W}(1.4-1.8 \mu \mathrm{m})$, and F222M $(2.15-2.30 \mu \mathrm{m})$. These filters are close to the standard ground-based $J, H, \& K$ filters. The observation of each cluster spanned three orbits of HST, with $\sim 42$ minutes of observing per orbit. This yielded total integration times of $1920 \mathrm{~s}$ in F110W, 3328s in F160W, and 2304s in F222M.

\section{Results}

Stellar photometry in crowded regions can be strongly affected by blending. Stephens et al. (2001) have studied the effects of blending on stellar photometry using three different techniques, including traditional completeness tests and the creation of completely artificial frames. These simulations are used to derive quantitative limits on where reliable photometry can be achieved. We use their results to reject measurements which may be affected by blending, and to correct for the effects of blending on the determination of the giant branch (GB) slope.

Individual cluster star measurements could only be extracted for the two less compact clusters G1 and G280. For these two clusters we perform statistical subtraction of the field to yield a cluster GB. To estimate the metallicity, we use the relationship between the GB slope $(\Delta(J-K) / \Delta(K))$ and globular cluster metallicity derived by Kuchinski et al. (1995) and Kuchinski \& Frogel (1995) for Galactic globular clusters.

The $M_{K^{-}}(J-K)$ CMDs of G280 are shown on the right side of Fig. 1. The left panel shows all the data inside a radius of $5^{\prime \prime}$, the radius chosen to define the cluster. Objects which are severly affected by blending $\left(\mu_{K}<14 \mathrm{mag} \operatorname{arcsec}^{-2}\right.$, $\left.r<1.0^{\prime \prime}\right)$ are plotted with half-size dots, and potential blends are plotted with open circles $\left(\mu_{K}<16, r<2.2^{\prime \prime}\right)$. The center panel shows objects measured outside the $5^{\prime \prime}$ cluster radius, which we attribute to the field. The right panel shows the result of statistically subtracting the field star component from the cluster (also omitting blends). At the top of the right panel is the linear fit to the GB. From this slope we estimate the blending-uncorrected metallicity of the G280 cluster as $+0.26 \pm 0.36$ dex. As previously mentioned, the measured slope, and thus the estimated metallicity, are affected by blending. Using the number - weighted average surface brightness of $\mu_{K}=16.9$ for the G280 cluster, we estimate a correction of $-0.42 \pm 0.09$ dex is required to remove the effects of blending. This gives a final metallicity of $-0.15 \pm 0.37$ dex for the G280 cluster.

For the purely infrared G1 cluster CMD we find a GB slope of $-0.083 \pm$ 0.014 , which implies a blending-uncorrected metallicity of $-1.00 \pm 0.42$ dex. Taking a number - weighted average surface brightness of $\mu_{K}=18.4$ for all usable cluster photometry, we estimate a blending correction of $-0.22 \pm 0.09$ dex to the cluster metallicity. This gives a final metallicity estimate of $-1.22 \pm 0.43$ dex for G1. We note however, that the range in our fitted luminosity $\left(\Delta M_{K} \sim\right.$ $2.5 \mathrm{mags}$ ) is only barely larger than half of the range used to define the relation ( $\Delta M_{K} \sim 4.5$ mags), slightly decreasing the confidence of our estimate.

We have also combined our infrared NICMOS data with the $V$-band WFPC2 observations of Rich et al. (1996) and Meylan et al. (2001). The resulting $M_{K_{0}}$ $(V-K)_{0}$ CMD is shown on the left side of Figure 1. In this diagram, the points indicate the mean $(V-K)_{0}$ obtained from both optical datasets. The 

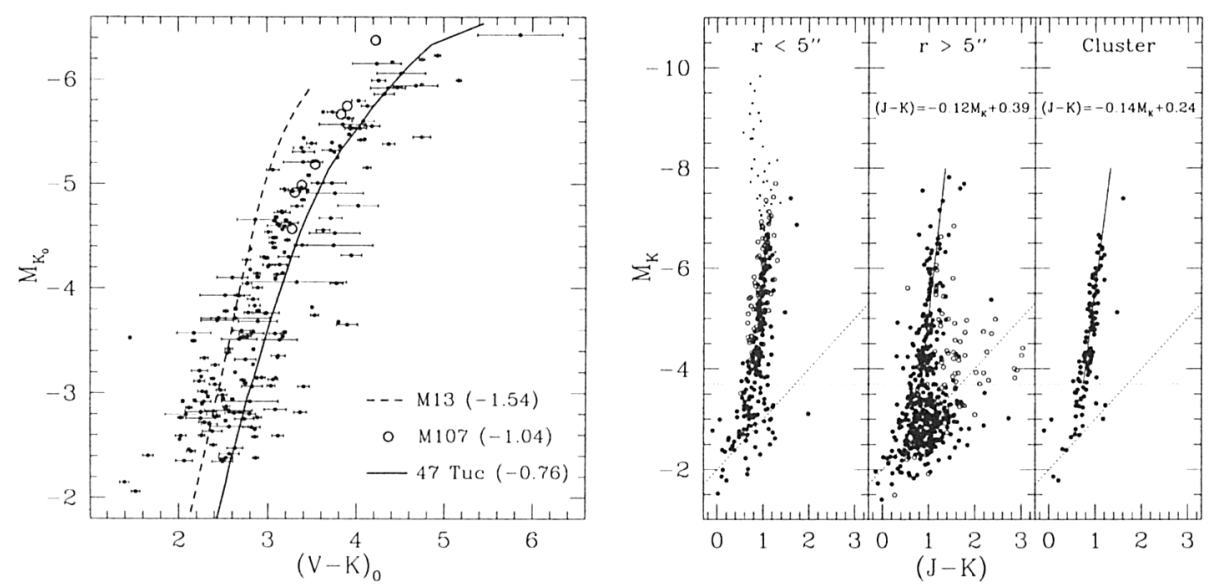

Figure 1. The left figure shows the G1 CMD created through the combination of NICMOS and WFPC2 data. The points are the mean $(V-K)_{0}$, and the errorbars illustrate the difference between two epochs of optical observations. This plot assumes $(m-M)_{0}=24.4, A_{K}=0.03$, and $E(V-K)=0.28$. The right plot shows the purely infrared CMD of the G280 cluster. Half size dots are blended objects, open circles indicate potentially blended objects. The right panel shows the cluster after statistical field subtraction.

errorbars illustrate the range of the observed $V$-band measurements. Since the measurement errors are relatively small, any large deviations are assumed to be indicative of stellar variability. Thus several of the most luminous stars near the top of the GB are undoubtedly variables. We have also over-plotted the RGB ridge lines of M13 and 47 Tucanae from Frogel et al. (1981), and stars in M107 measured by Frogel et al. (1983). The G1 RGB position and morphology in this CMD indicates that G1 probably has a slightly higher metallicity $([\mathrm{Fe} / \mathrm{H}] \sim-0.9$ dex) than we obtained from the slope of the purely infrared GB.

\section{References}

Frogel, J.A., Persson, S.E., \& Cohen, J.G. 1981 ApJ, 246, 842

Frogel, J.A., Persson, S.E., \& Cohen, J.G. 1983 ApJS, 53, 713

Kuchinski, L.E. \& Frogel, J.A. 1995 AJ, 110, 2844

Kuchinski, L.E., Frogel, J.A., Terndrup, D.M., \& Persson, S.E. 1995 AJ, 109, 1131

Meylan, G., Sarajedini, A., Jablonka, P., Djorgovski, S. G., Bridges, T., \& Rich, R. M. 2001, AJ, 122, 830

Rich, R.M., Mighell, K.J., Freedman, W.L. \& Neill, J.D. 1996 AJ, 111, 768

Stephens, A.W., Frogel, J.A., Freedman, W., Gallart, C., Jablonka, P., Ortolani, S., Renzini, A., Rich, R.M. \& Davies, R. 2001, AJ, 121, 2584 